\title{
A convenient procedure for bismuth-mediated Barbier-type allylation of aldehydes in water containing fluoride ions
}

\author{
Keith Smith, ${ }^{* a}$ Sandra Lock, ${ }^{a}$ Gamal A. El-Hiti, $\grave{\dagger}^{a}$ Makoto Wada ${ }^{b}$ and Norikazu Miyoshi ${ }^{b}$ \\ ${ }^{a}$ Centre for Clean Chemistry, Department of Chemistry, University of Wales Swansea, \\ Singleton Park, Swansea, UK SA2 8PP \\ ${ }^{b}$ Department of Chemistry, Faculty of Integrated Arts and Sciences, University of Tokushima, \\ Minami-Josanjima, Tokushima, Japan 770
}

Received 8th January 2004, Accepted 11th February 2004

First published as an Advance Article on the web 25th February 2004

A new and convenient procedure for synthesis of homoallylic alcohols in generally good to excellent yields has been developed. The bismuth-mediated Barbier-type allylation of aldehydes (aromatic, aliphatic, alicyclic and heterocyclic) with allyl bromide has been carried out smoothly in water in the presence of fluoride ions.

\section{Introduction}

Traditionally performed in organic solvents with the strict exclusion of air and moisture, the coupling of an organic halide and a carbonyl compound in the presence of a metal (the Barbier reaction) is a fundamental procedure for making new carbon-carbon bonds. ${ }^{1}$ Interest in the reaction surged in the early 1980's with the discovery that allylic halides in particular reacted with aldehydes in the presence of a range of metals in aqueous media, sometimes with the assistance of dissolved salts, organic co-solvents or sonication to boost reactivity. ${ }^{2}$ Attendant benefits included the ability to dispense with timeconsuming drying regimes and protection-deprotection steps when dealing with vulnerable substrates, but recently, with the current emphasis on developing cleaner, safer, more atomefficient chemical processes that depend less on organic solvents, ${ }^{3}$ aqueous Barbier-type procedures have acquired a new relevance.

Bismuth is recognised alongside tin, zinc and indium metals as an established and successful mediator of aqueous Barbiertype reactions, ${ }^{4,5}$ though it is usually prepared in situ from a bismuth salt and a reducing metal in water/THF solvent mixtures. ${ }^{6}$ The toxicity of bismuth is considered to be low; ${ }^{7,8}$ indeed, bismuth salts have, for a long time, been used in cosmetics and anti-viral creams and are important components of orally administered gastrointestinal treatments currently prescribed on a worldwide basis. ${ }^{8}$ Accordingly, the metal does not seem to have the degree of toxicity that makes the use of tin and its compounds, for instance, much less favourable in organic synthesis. ${ }^{5}$ Zinc is commonly accepted to be non-toxic and is usually activated in aqueous Barbier-type allylations by the addition of ammonium chloride. However, with some notable exceptions, ${ }^{9-12}$ it generally requires the presence of an organic cosolvent to achieve high product yields. ${ }^{13-17}$ In contrast, indium is distinguished by its versatility and ability to mediate reactions quickly and smoothly in water alone ${ }^{2}$ without the need for an additional means of activation. On a mole for mole basis, however, it is by far the most expensive of the four metals.

Bismuth powder is readily available and easy to use and allylations of a range of aldehydes in DMF and acetonitrile have been published. ${ }^{6,18,19}$ However, in a recent paper that compared the merits of bismuth, indium, copper and zinc powders in temperature-controlled solvent-free allylations, the reported results indicated that while bismuth provided worthwhile

$\dagger$ Permanent address: Department of Chemistry, Faculty of Science, Tanta University, Tanta 31527, Egypt. conversions of benzaldehyde and salicylaldehyde, the metal may otherwise have limited general capability when no solvent is present. ${ }^{20}$ Very recently, the bismuth-mediated Barbier-type allylation of carbonyl compounds in the absence of solvent, affording homoallyl alcohols in good yield, was reported. ${ }^{21}$ However, the method involves the use of a ball milling technique and a large excess of bismuth (aldehyde : Bi ratio is $1: 8)$. Moreover, the reaction has been applied only on a very small scale $(0.25 \mathrm{mmol}$ of aldehyde).

As part of our continuing interest in the development of clean chemical processes, ${ }^{22,23}$ we became interested in aqueous bismuth-mediated reactions as a possible method for the synthesis of homoallylic alcohols. We therefore wish to disclose the results of our own bismuth-mediated allylations performed in entirely aqueous solutions.

\section{Results and discussion}

As a test case, the allylation reaction of benzaldehyde (1a) with allyl bromide (2) in the presence of bismuth powder in water (eqn. (1)) was investigated.

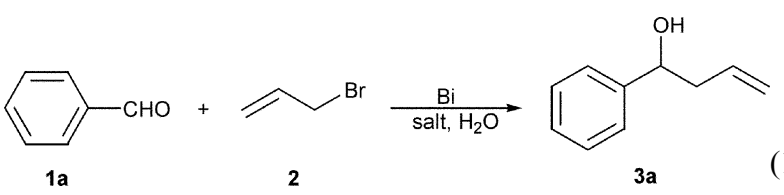

Keeping similar mole ratios of reactants as were previously used with $\mathrm{DMF}^{18}$ but changing the solvent to distilled water, we began by stirring benzaldehyde (1a) $(1 \mathrm{mmol})$ and allyl bromide (2) $(1.16 \mathrm{mmol})$ with bismuth powder $(1.2 \mathrm{mmol})$ in distilled water $(5 \mathrm{ml})$ for $24 \mathrm{~h}$ at room temperature under a static argon atmosphere. This resulted in a yield of 1-phenylbut-3-en-1-ol (3a) of only $31 \%$ (by GC). The performance of zinc in aqueous Barbier-type reactions is enhanced by dissolved ammonium chloride and recent reports indicate that fluoride salts successfully activate carbon-carbon bond-forming reactions mediated by antimony and aluminium in aqueous solution. ${ }^{24,25}$ Therefore, we investigated the effect of a variety of additives and mole ratios of reactants, this time beginning with a similar mole ratio to that adopted by $\mathrm{Li}$ et al. $^{24}$ in Sb-mediated allylation reactions. The results are summarized in Table 1.

Compared to our first experiment in water, the yield of homoallylic alcohol did not improve, and was, in fact, somewhat depressed in the presence of the larger excesses of bismuth powder and allyl bromide (Entry 1, Table 1). Various salts were found to improve the yield, and amongst the fluoride and 
Table 1 Synthesis of 1-phenylbut-3-en-1-ol (3a), according to equation (1), ${ }^{a}$ in the presence of various salts

\begin{tabular}{|c|c|c|c|c|}
\hline Entry & $\mathrm{Bi}(\mathrm{mmol})$ & Allyl bromide (mmol) & Salt (mmol) & Yield $(\%)^{b}$ \\
\hline 1 & 5 & 2.3 & - & $20^{c}$ \\
\hline 2 & 5 & 2.3 & $(\text { sonication } 3 \mathrm{~h})^{d}$ & $52^{c}$ \\
\hline 3 & 5 & 2.3 & $\mathrm{NH}_{4} \mathrm{Cl}(10)$ & $18^{c}$ \\
\hline 4 & 5 & 2.3 & $\mathrm{NaCl}(10)$ & 54 \\
\hline 5 & 5 & 2.3 & $\mathrm{KF}(10)$ & 83 \\
\hline 6 & 2 & 2.3 & KF (10) & 82 \\
\hline 7 & 2 & 2.3 & $\mathrm{NaF}(10)$ & 40 \\
\hline 8 & 2 & 2.3 & $\mathrm{KF}(5)$ & 90 \\
\hline 9 & 2 & 2.3 & $\mathrm{NaF}(5)$ & 59 \\
\hline 10 & 2 & 2.3 & $\operatorname{LiF}(5)$ & 58 \\
\hline 11 & 1.1 & 2.3 & $\mathrm{KF}(5)$ & 89 \\
\hline 12 & 1.1 & 1.16 & $\mathrm{KF}(5)$ & $>99^{e}$ \\
\hline 13 & 1.1 & 1.16 & $\mathrm{KF}(1.1)$ & $>99$ \\
\hline 14 & 1.1 & 1.16 & $\mathrm{KF}(0.5)$ & 83 \\
\hline
\end{tabular}

${ }^{a}$ A mixture of benzaldehyde $(106.2 \mathrm{mg}, 1 \mathrm{mmol})$, allyl bromide, Bi powder and $\mathrm{KF}$ in water $(5 \mathrm{ml})$ was stirred for $12 \mathrm{~h}$ at room temperature.

${ }^{b}$ Determined by quantitative GC analysis. ${ }^{c}$ Reaction time 24 h. ${ }^{d}$ Using a Decon ${ }^{\circledR}$ FS100B $47 \mathrm{KHz}$ ultrasonic cleaning bath. ${ }^{e}$ Reaction complete in $3 \mathrm{~h}$.

chloride salts tested, potassium fluoride proved to be the most effective. As the excesses of $\mathrm{Bi}$ and/or the allyl bromide were reduced, the yield of the desired product increased. When $\mathrm{Bi}$ and the allyl bromide were each in only slight excess over 1 mole per mole of benzaldehyde, the yield was almost quantitative even after 3 hours (Entry 12).

Furthermore, the amount of KF could be reduced to 1.1 mmol and still enabled a quantitative yield within 12 hours (Entry 13). Even with only 0.5 mole equivalents of KF, $83 \%$ of the desired alcohol was achieved in 12 hours (Entry 14). This suggests that the relationship between the potassium fluoride and bismuth is not a stoichiometric one. However, reactions involving different amounts of bismuth but constant amounts of benzaldehyde $(1.0 \mathrm{mmol})$, allyl bromide $(1.16 \mathrm{mmol})$, and potassium fluoride $(5 \mathrm{mmol})$ suggest a $1: 1$ metal : allyl dependence (Fig. 1).

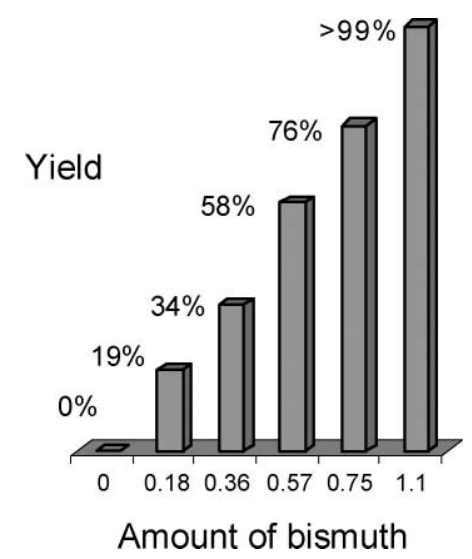

Fig. 1 Effect of amount of Bi upon the GC yield of 3a in the reaction of $1 \mathrm{a}(1 \mathrm{mmol})$ and $2(1.16 \mathrm{mmol})$ in aq. $\operatorname{KF}(1 \mathrm{M}, 5 \mathrm{ml})$ for $12 \mathrm{~h}$ at room temperature.

The generality of the method was investigated with a range of aldehydes 1 (4-tolualdehyde, 3-bromobenzaldehyde, 4-nitrobenzaldehyde, 2-hydroxybenzaldehyde, 2-naphthaldehyde, 2furaldehyde, cinnamaldehyde, 3-phenylpropanal, 2-phenylpropanal, octanal, cyclohexanecarboxaldehyde), using allyl bromide $(1.16 \mathrm{mmol}), \mathrm{Bi}(1.1 \mathrm{mmol})$ and potassium fluoride $(5$ mmol, unless otherwise stated), according to eqn. (2), to afford allylic alcohols 3 in excellent yields (Table 2).

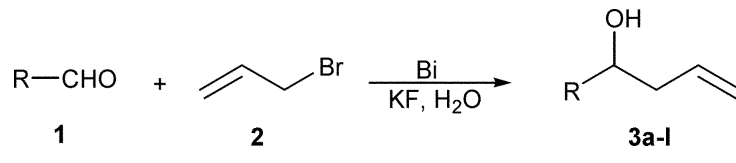

Table 2 Synthesis of $\mathbf{3}$ according to equation (2) ${ }^{a}$

\begin{tabular}{|c|c|c|c|}
\hline Entry & Product & $\mathrm{R}$ & Yield $(\%)^{b}$ \\
\hline 1 & $\mathbf{3 a}$ & $\mathrm{C}_{6} \mathrm{H}_{5}$ & 99 \\
\hline 2 & $\mathbf{3 b}$ & $4-\mathrm{MeC}_{6} \mathrm{H}_{4}$ & $99(47)^{c}$ \\
\hline 3 & $3 c$ & $3-\mathrm{BrC}_{6} \mathrm{H}_{4}$ & $90(28)^{c}$ \\
\hline 4 & 3d & $4-\mathrm{O}_{2} \mathrm{NC}_{6} \mathrm{H}_{4}$ & $62(84)^{d}(86)^{e}$ \\
\hline 5 & $3 e$ & $2-\mathrm{HOC}_{6} \mathrm{H}_{4}$ & 86 \\
\hline 6 & $3 f$ & 2-naphthyl & $94^{f}$ \\
\hline 7 & 3g & 2-furyl & 91 \\
\hline 8 & $3 \mathbf{h}$ & $\mathrm{C}_{6} \mathrm{H}_{5} \mathrm{CH}=\mathrm{CH}$ & 94 \\
\hline 9 & $3 \mathbf{i}$ & $\mathrm{C}_{6} \mathrm{H}_{5} \mathrm{CH}_{2} \mathrm{CH}_{2}$ & 80 \\
\hline 10 & $3 \mathbf{j}$ & $\mathrm{C}_{6} \mathrm{H}_{5} \mathrm{CH}\left(\mathrm{CH}_{3}\right)$ & 93 \\
\hline 11 & $3 \mathbf{k}$ & ${ }^{n} \mathrm{C}_{7} \mathrm{H}_{15}$ & 91 \\
\hline 12 & 31 & cyclohexyl & 99 \\
\hline
\end{tabular}

${ }^{a}$ A mixture of aldehyde (1.0 mmol), allyl bromide (1.16 mmol), Bi (1.1 $\mathrm{mmol})$ and $\mathrm{KF}(5 \mathrm{mmol})$ in water $(5 \mathrm{ml})$ was stirred at room temperature for 12 h. ${ }^{b}$ Determined by quantitative GC analysis. ${ }^{c}$ Using KF (1.1 mmol). ${ }^{d}$ Using KF (7.5 mmol). ${ }^{e}$ Using KF $(10.0 \mathrm{mmol}) .{ }^{f}$ Isolated yield.

Benzaldehyde (Entry 1, Table 2) was transformed into an essentially quantitative yield of $\mathbf{3 a}$, with just small traces of benzyl alcohol and benzoic acid as by-products. Hydrobenzoin, the pinacol coupling product, was not detected. Allylations of the majority of other aromatic aldehydes examined also proceeded smoothly in water containing $5 \mathrm{mmol}$ of potassium fluoride within the $12 \mathrm{~h}$ period to afford good to excellent yields of the corresponding homoallylic alcohols. However, although 4-tolualdehyde and 3-bromobenzaldehyde gave excellent yields ( 99 and $90 \%$ of $\mathbf{3 b}$ and $\mathbf{3 c}$, respectively) under these standard conditions, they reacted more sluggishly and gave poorer yields ( 47 and $28 \%$, respectively) when the amount of KF was reduced to $1.1 \mathrm{mmol}$ (Entries 2 and 3, Table 2). 4-Nitrobenzaldehyde gave only a modest yield $(62 \%)$ under the standard conditions, but responded satisfactorily, giving a yield of $86 \%$, when the amount of fluoride salt was increased to $10 \mathrm{mmol}$ (Entry 4, Table 2). Thus, the reaction is quite general for a range of substituted aromatic aldehydes.

In order to assess the usefulness of the reaction with other types of aldehydes, reactions were conducted with a heterocyclic aldehyde, 2-furaldehyde (Entry 7, Table 2), an $\alpha \beta$-unsaturated aldehyde, cinnamaldehyde (Entry 8, Table 2), various aliphatic aldehydes (3-phenylpropanal; 2-phenylpropanal; octanal; Entries 9-11, Table 2) and an alicyclic aldehyde, cyclohexanecarboxaldehyde (Entry 12, Table 2). In all cases a high yield of the corresponding homoallylic alcohol was obtained (Table 2). The new method therefore appears to be quite general. 


\section{Conclusion}

A new method for allylation of aldehydes, involving use of bismuth metal and allyl bromide in water containing $\mathrm{KF}$, has been developed. The method affords excellent yields of homoallylic alcohols. Unfortunately, from a green chemistry viewpoint a reaction using potassium fluoride is not ideal. Nevertheless the reaction has demonstrated that there are circumstances in which it is possible to dispense completely with the usual organic solvent (THF) yet still retain useful product yields. Therefore, there is still room for considerable improvement in the clean synthesis of allylic alcohols. We continue to investigate other synthetic capabilities of bismuth in water.

\section{Experimental}

Commercially available starting materials (Aldrich) were used as supplied. GC was carried out using a PU 4400 Gas Chromatograph (Philips) fitted with a carbowax capillary column $(15 \mathrm{~m} \times 0.32 \mathrm{~mm}$ id). Hexadecane was added as an internal standard to allow quantification. Infrared spectra were recorded on a Horiba FT-IR 210 spectrophotometer. ${ }^{1} \mathrm{H}$ and ${ }^{13} \mathrm{C}$ NMR spectra were recorded on a Bruker AC-400 Fourier Transform spectrometer operating at $400 \mathrm{MHz}$ for ${ }^{1} \mathrm{H}$ and 100 $\mathrm{MHz}$ for ${ }^{13} \mathrm{C}$ measurement. Chemical shifts are reported relative to tetramethylsilane. Assignments of signals are based on coupling patterns and expected chemical shift values and have not been rigorously confirmed. Signals with similar characteristics might be interchanged. Flash column chromatography was performed using Merck Matrix Silica 60 (35-70 micron) silica gel.

\section{Typical reaction procedure}

Bismuth powder $(231.8 \mathrm{mg}, 1.1 \mathrm{mmol})$ was weighed into a $50 \mathrm{ml}$ two-necked flask equipped with a magnetic stirrer bar and capped with a septum. The vessel was flushed with argon for 5 min and aq. KF solution ( $1 \mathrm{M}, 5 \mathrm{ml})$ was added. The argon flow was stopped and allyl bromide $(0.10 \mathrm{ml}, 1.16 \mathrm{mmol})$ and aldehyde $(1.0 \mathrm{mmol})$ were added in quick succession. The mixture was stirred for $12 \mathrm{~h}$ at room temperature and the organic materials were then extracted with diethyl ether $(3 \times 20 \mathrm{ml})$. The combined extract was washed with aq. $\mathrm{NaCl}$ solution $(2 \times 15$ $\mathrm{ml}$ ) and dried over anhydrous $\mathrm{MgSO}_{4}$. The organic phase was filtered and evaporated to leave oily residue, which was purified by flash column chromatography on silica gel using hexaneEtOAc $(4: 1)$ as the solvent to give pure 3 , which was analysed by GC. Confirmation of the product was achieved by use of NMR and by comparison with literature values. ${ }^{21,26-32}$

\section{1-Phenylbut-3-en-1-ol (3a) ${ }^{26-29}$}

Oil, $\delta_{\mathrm{H}}\left(\mathrm{CDCl}_{3}\right) 7.36-7.25(\mathrm{~m}, 5 \mathrm{H}, \mathrm{ArH}), 5.74-5.33(\mathrm{~m}, 1 \mathrm{H}$, $\left.\mathrm{CH}=\mathrm{CH}_{2}\right), 5.09-5.00\left(\mathrm{~m}, 2 \mathrm{H}, \mathrm{CH}=\mathrm{CH}_{2}\right), 4.61(\mathrm{t}, J=6 \mathrm{~Hz}, 1 \mathrm{H}$, $\mathrm{CHOH}), 2.43-2.38\left(\mathrm{~m}, 2 \mathrm{H}, \mathrm{CH}_{2}\right), 2.15$ (br s, exch., $1 \mathrm{H}, \mathrm{OH}$ ); $\delta_{\mathrm{C}}\left(\mathrm{CDCl}_{3}\right) 144.8(\mathrm{~s}), 134.9\left(\mathrm{~d}, \mathrm{CH}=\mathrm{CH}_{2}\right), 128.8(\mathrm{~d}), 128.0(\mathrm{~d})$, 126.3 (d), 118.8 (t, $\left.\mathrm{CH}=\mathrm{CH}_{2}\right), 73.8(\mathrm{~d}, \mathrm{CHOH}), 44.2\left(\mathrm{t}, \mathrm{CH}_{2}\right)$.

\section{1-(4-Methylphenyl)but-3-en-1-ol (3b) $)^{26,27}$}

Oil, $\delta_{\mathrm{H}}\left(\mathrm{CDCl}_{3}\right) 7.18(\mathrm{~d}, J=8 \mathrm{~Hz}, 2 \mathrm{H}, \mathrm{ArH}), 7.09$ (d, $J=8 \mathrm{~Hz}$, $2 \mathrm{H}, \mathrm{ArH}), 5.79-5.68\left(\mathrm{~m}, 1 \mathrm{H}, \mathrm{CH}=\mathrm{CH}_{2}\right), 5.12-5.04(\mathrm{~m}, 2 \mathrm{H}$, $\left.\mathrm{CH}=\mathrm{CH}_{2}\right), 4.63(\mathrm{t}, J=6 \mathrm{~Hz}, 1 \mathrm{H}, \mathrm{CHOH}), 2.45-2.41(\mathrm{~m}, 2 \mathrm{H}$, $\left.\mathrm{CH}_{2}\right), 2.27$ (s, $\left.3 \mathrm{H}, \mathrm{CH}_{3}\right), 1.94$ (br s, exch., $\left.1 \mathrm{H}, \mathrm{OH}\right) ; \delta_{\mathrm{C}}\left(\mathrm{CDCl}_{3}\right)$ 141.3 (s), 137.6 (s), 135.0 (d, $\mathrm{CH}=\mathrm{CH}_{2}$ ), 129.5 (d), 126.2 (d), $118.7\left(\mathrm{t}, \mathrm{CH}=\mathrm{CH}_{2}\right), 73.6(\mathrm{~d}, \mathrm{CHOH}), 44.2\left(\mathrm{t}, \mathrm{CH}_{2}\right), 21.5(\mathrm{q}$, $\left.\mathrm{CH}_{3}\right)$.

\section{1-(3-Bromophenyl)but-3-en-1-ol (3c) ${ }^{30}$}

Oil, $\delta_{\mathrm{H}}\left(\mathrm{CDCl}_{3}\right) 7.45(\mathrm{~d}, J=1.2 \mathrm{~Hz}, 1 \mathrm{H}, \mathrm{ArH}), 7.33(\mathrm{dd}, J=7.2$, 1.2 Hz, 1 H, ArH), 7.21-7.11 (m, 2 H, ArH), 5.77-5.65 (m, $1 \mathrm{H}$,
$\left.\mathrm{C} H=\mathrm{CH}_{2}\right), 5.13-5.06\left(\mathrm{~m}, 2 \mathrm{H}, \mathrm{CH}=\mathrm{CH}_{2}\right), 4.62(\mathrm{t}, J=7 \mathrm{~Hz}, 1 \mathrm{H}$, $\mathrm{CHOH}), 2.48-2.32$ (m, $2 \mathrm{H}, \mathrm{CH}_{2}$ ), 2.24 (br s, exch., $1 \mathrm{H}, \mathrm{OH}$ ); $\delta_{\mathrm{C}}\left(\mathrm{CDCl}_{3}\right) 146.6(\mathrm{~s}), 134.3\left(\mathrm{~d}, \mathrm{CH}=\mathrm{CH}_{2}\right), 131.0(\mathrm{~d}), 130.4(\mathrm{~d})$, 129.3 (d), 124.9 (d), 123.0 (s), 119.4 (t, $\mathrm{CH}=\mathrm{CH}_{2}$ ), 72.9 (d, $\mathrm{CHOH}), 44.2\left(\mathrm{t}, \mathrm{CH}_{2}\right)$.

\section{1-(4-Nitrophenyl)but-3-en-1-ol (3d) ${ }^{21,27,29}$}

Oil, $\delta_{\mathrm{H}}\left(\mathrm{CDCl}_{3}\right) 8.12(\mathrm{~d}, J=8.7 \mathrm{~Hz}, 2 \mathrm{H}, \mathrm{ArH}), 7.46(\mathrm{~d}$, $J=8.7 \mathrm{~Hz}, 2 \mathrm{H}, \mathrm{ArH}), 5.76-5.66\left(\mathrm{~m}, 1 \mathrm{H}, \mathrm{CH}=\mathrm{CH}_{2}\right), 5.14-5.07$ $\left(\mathrm{m}, 2 \mathrm{H}, \mathrm{CH}=\mathrm{CH}_{2}\right), 4.79(\mathrm{dd}, J=8.9,4.7 \mathrm{~Hz}, 1 \mathrm{H}, \mathrm{CHOH})$, 2.52-2.34 (m, $\left.2 \mathrm{H}, \mathrm{CH}_{2}\right), 1.52$ (br s, exch., $\left.1 \mathrm{H}, \mathrm{OH}\right) ; \delta_{\mathrm{C}}\left(\mathrm{CDCl}_{3}\right)$ 151.6 (s), 147.6 (s), 133.6 (d, $\left.\mathrm{CH}=\mathrm{CH}_{2}\right), 127.0$ (d), 124.0 (d), $120.0\left(\mathrm{t}, \mathrm{CH}=\mathrm{CH}_{2}\right), 72.6(\mathrm{~d}, \mathrm{CHOH}), 44.3\left(\mathrm{t}, \mathrm{CH}_{2}\right)$.

\section{1-(2-Hydroxyphenyl)but-3-en-1-ol (3e $)^{31}$}

Oil, $\delta_{\mathrm{H}}\left(\mathrm{CDCl}_{3}\right) 7.99$ (s, exch., $\left.1 \mathrm{H}, \mathrm{OH}\right), 7.15-6.74(\mathrm{~m}, 4 \mathrm{H}$, $\mathrm{ArH})$, 5.84-5.73 (m, $\left.1 \mathrm{H}, \mathrm{CH}=\mathrm{CH}_{2}\right), 5.19-5.12(\mathrm{~m}, 2 \mathrm{H}$, $\left.\mathrm{CH}=\mathrm{CH}_{2}\right), 4.80(\mathrm{t}, J=6.6 \mathrm{~Hz}, 1 \mathrm{H}, \mathrm{CHOH}), 2.67$ (br s, exch., $1 \mathrm{H}, \mathrm{OH}), 2.60-2.48\left(\mathrm{~m}, 2 \mathrm{H}, \mathrm{CH}_{2}\right) ; \delta_{\mathrm{C}}\left(\mathrm{CDCl}_{3}\right) 155.9$ (s), 134.3 (d, $\mathrm{CH}=\mathrm{CH}_{2}$ ), 129.4 (d), 127.5 (d), 126.5 (s), 120.2 (d), 119.9 $\left(\mathrm{t}, \mathrm{CH}=\mathrm{CH}_{2}\right), 117.7$ (d), $75.1(\mathrm{~d}, \mathrm{CHOH}), 42.6\left(\mathrm{t}, \mathrm{CH}_{2}\right)$.

\section{1-(2-Naphthyl)but-3-en-1-ol (3f) ${ }^{32}$}

Oil, $\delta_{\mathrm{H}}\left(\mathrm{CDCl}_{3}\right)$ 7.77-7.71 (m, $\left.4 \mathrm{H}, \mathrm{ArH}\right), 7.42-7.36(\mathrm{~m}, 3 \mathrm{H}$, ArH), 5.81-5.69 (m, $\left.1 \mathrm{H}, \mathrm{CH}=\mathrm{CH}_{2}\right), 5.14-5.05(\mathrm{~m}, 2 \mathrm{H}$, $\left.\mathrm{CH}=\mathrm{CH}_{2}\right), 4.82(\mathrm{dd}, J=7.4,5.4 \mathrm{~Hz}, 1 \mathrm{H}, \mathrm{CHOH}), 2.57-2.45$ $\left(\mathrm{m}, 2 \mathrm{H}, \mathrm{CH}_{2}\right), 2.12$ (br s, exch., $\left.1 \mathrm{H}, \mathrm{OH}\right) ; \delta_{\mathrm{C}}\left(\mathrm{CDCl}_{3}\right) 141.7$ (s), 134.9 (d, $\left.\mathrm{CH}=\mathrm{CH}_{2}\right), 133.7$ (s), 133.4 (s), 128.7 (d), 128.5 (d), 128.4 (d), 126.6 (d), 126.3 (d), 125.0 (d), 124.5 (d), 118.9 (t, $\left.\mathrm{CH}=\mathrm{CH}_{2}\right), 73.9(\mathrm{~d}, \mathrm{CHOH}), 44.1\left(\mathrm{t}, \mathrm{CH}_{2}\right)$.

\section{1-(2-Furyl)but-3-en-1-ol (3g) 26}

Oil, $\delta_{\mathrm{H}}\left(\mathrm{CDCl}_{3}\right) 7.31$ (dd, $J=1.8,0.8 \mathrm{~Hz}, 1 \mathrm{H}$, furyl), 6.26 (dd, $J=3.2,1.8 \mathrm{~Hz}, 1 \mathrm{H}$, furyl), 6.18 (dd, $J=3.2,0.8 \mathrm{~Hz}, 1 \mathrm{H}$, furyl), 5.79-5.68 (m, $\left.1 \mathrm{H}, \mathrm{CH}=\mathrm{CH}_{2}\right), 5.15-5.06\left(\mathrm{~m}, 2 \mathrm{H}, \mathrm{CH}=\mathrm{CH}_{2}\right)$, $4.68(\mathrm{t}, J=6.5 \mathrm{~Hz}, 1 \mathrm{H}, \mathrm{CHOH}), 2.63-2.49\left(\mathrm{~m}, 2 \mathrm{H}, \mathrm{CH}_{2}\right), 2.05$ (br s, exch., $1 \mathrm{H}, \mathrm{OH}) ; \delta_{\mathrm{C}}\left(\mathrm{CDCl}_{3}\right) 156.4(\mathrm{~s}), 142.4(\mathrm{~d}), 134.1$ (d, $\mathrm{CH}=\mathrm{CH}_{2}$ ), 119.0 (t, $\mathrm{CH}=\mathrm{CH}_{2}$ ), 110.6 (d), 106.5 (d), 67.3 (d, $\mathrm{CHOH}), 40.5\left(\mathrm{t}, \mathrm{CH}_{2}\right)$.

\section{1-Phenylhexa-1,5-dien-3-ol (3h) ${ }^{27,29,31}$}

Oil, $\delta_{\mathrm{H}}\left(\mathrm{CDCl}_{3}\right) 7.31(\mathrm{~d}, J=7.2 \mathrm{~Hz}, 2 \mathrm{H}, \mathrm{ArH}), 7.24(\mathrm{t}$, $J=7.2 \mathrm{~Hz}, 2 \mathrm{H}, \mathrm{ArH}), 7.17(\mathrm{~m}, 1 \mathrm{H}, \mathrm{ArH}), 6.53(\mathrm{~d}, J=15.9 \mathrm{~Hz}$, $1 \mathrm{H}, \mathrm{CH}), 6.17(\mathrm{dd}, J=15.9,6.4 \mathrm{~Hz}, 1 \mathrm{H}, \mathrm{CH}), 5.83-5.73$ $\left(\mathrm{m}, 1 \mathrm{H}, \mathrm{CH}=\mathrm{CH}_{2}\right), 5.15-5.08\left(\mathrm{~m}, 2 \mathrm{H}, \mathrm{CH}=\mathrm{CH}_{2}\right), 4.29(\mathrm{t}$, $J=6.4 \mathrm{~Hz}, 1 \mathrm{H}, \mathrm{CHOH}), 2.41-2.27\left(\mathrm{~m}, 2 \mathrm{H}, \mathrm{CH}_{2}\right), 1.82(\mathrm{br} \mathrm{s}$, exch., $1 \mathrm{H}, \mathrm{OH}) ; \delta_{\mathrm{C}}\left(\mathrm{CDCl}_{3}\right) 137.0(\mathrm{~s}), 134.4\left(\mathrm{~d}, \mathrm{CH}=\mathrm{CH}_{2}\right), 131.9$ (d), 130.2 (d), 129.0 (d), 128.1 (d), 126.9 (d), $119.0\left(\mathrm{t}, \mathrm{CH}=\mathrm{CH}_{2}\right)$, 72.1 (d, $\mathrm{CHOH}), 42.4\left(\mathrm{t}, \mathrm{CH}_{2}\right)$.

\section{6-Phenylhex-1-en-4-ol (3i) ${ }^{28}$}

Oil, $\delta_{\mathrm{H}}\left(\mathrm{CDCl}_{3}\right) 7.18-7.03(\mathrm{~m}, 5 \mathrm{H}, \mathrm{ArH}), 5.76-5.62(\mathrm{~m}, 1 \mathrm{H}$, $\left.\mathrm{CH}=\mathrm{CH}_{2}\right), 5.05-4.98\left(\mathrm{~m}, 2 \mathrm{H}, \mathrm{CH}=\mathrm{CH}_{2}\right), 3.59-3.51(\mathrm{~m}, 1 \mathrm{H}$, $\mathrm{CHOH}), 2.74-2.51\left(\mathrm{~m}, 2 \mathrm{H}, \mathrm{CH}_{2}\right), 2.23-2.01\left(\mathrm{~m}, 2 \mathrm{H}, \mathrm{CH}_{2}\right)$, 1.81 (br s, exch., $1 \mathrm{H}, \mathrm{OH}), 1.70-1.62\left(\mathrm{~m}, 2 \mathrm{H}, \mathrm{CH}_{2}\right) ; \delta_{\mathrm{C}}\left(\mathrm{CDCl}_{3}\right)$ 142.5 (s), 135.0 (d, $\left.\mathrm{CH}=\mathrm{CH}_{2}\right), 128.8$ (d), 128.7 (d), 126.3 (d), $118.8\left(\mathrm{t}, \mathrm{CH}=\mathrm{CH}_{2}\right), 70.4(\mathrm{~d}, \mathrm{CHOH}), 42.5\left(\mathrm{t}, \mathrm{CH}_{2}\right), 38.9(\mathrm{t}$, $\left.\mathrm{CH}_{2}\right), 32.5\left(\mathrm{t}, \mathrm{CH}_{2}\right)$.

\section{5-Phenylhex-1-en-4-ol (3j)}

Oil, mixture of syn and anti diastereoisomers in almost equal proportions, $\delta_{\mathrm{H}}\left(\mathrm{CDCl}_{3}\right) 7.39-7.22(\mathrm{~m}, 5 \mathrm{H}, \mathrm{ArH}), 5.98-5.78(\mathrm{~m}$, $\left.1 \mathrm{H}, \mathrm{CH}=\mathrm{CH}_{2}\right), 5.20-5.10\left(\mathrm{~m}, 2 \mathrm{H}, \mathrm{CH}=\mathrm{CH}_{2}\right), 3.81-3.73(\mathrm{~m}$, $1 \mathrm{H}, \mathrm{CHOH}), 2.88-2.78\left(\mathrm{~m}, 1 \mathrm{H}, \mathrm{CHCH}_{3}\right), 2.47-2.02(\mathrm{~m}, 2 \mathrm{H}$, $\mathrm{CH}_{2}$ ), 1.78 (br s, exch., $\left.1 \mathrm{H}, \mathrm{OH}\right), 1.39,1.34$ (2 dd, $J=7.0$, $\left.1.3 \mathrm{~Hz}, 3 \mathrm{H}, \mathrm{CH}_{3}\right) ; \delta_{\mathrm{C}}\left(\mathrm{CDCl}_{3}\right) 144.9,143.8(2 \mathrm{~s}, \mathrm{C}-1$ of $\mathrm{Ph})$, 
$135.6,135.5\left(2 \mathrm{~d}, \mathrm{CH}=\mathrm{CH}_{2}\right), 128.9,128.7,128.2,127.9(4 \mathrm{~d}, \mathrm{C}-2$ and $\mathrm{C}-3$ of $\mathrm{Ph}$ ), 127.1, 126.9 (2 d, C-4 of Ph), 118.4, 118.1 (2 t, $\left.\mathrm{CH}=\mathrm{CH}_{2}\right), 75.6,75.5(2 \mathrm{~d}, \mathrm{CHOH}), 45.8,45.7\left(2 \mathrm{t}, \mathrm{CH}_{2}\right), 39.9$, 39.4 ( $\left.2 \mathrm{~d}, \mathrm{CHCH}_{3}\right), 18.2,16.9\left(2 \mathrm{q}, \mathrm{CH}_{3}\right)$.

\section{Undec-1-en-4-ol (3k) ${ }^{29}$}

Oil, $\delta_{\mathrm{H}}\left(\mathrm{CDCl}_{3}\right)$ 5.82-5.71 (m, $\left.1 \mathrm{H}, \mathrm{CH}=\mathrm{CH}_{2}\right), 5.10-5.04(\mathrm{~m}$, $\left.2 \mathrm{H}, \mathrm{CH}=\mathrm{CH}_{2}\right), 3.61-3.53(\mathrm{~m}, 1 \mathrm{H}, \mathrm{CHOH}), 2.28-2.02(\mathrm{~m}, 2 \mathrm{H}$ $\mathrm{CH}_{2}$ ), 1.80 (br s, exch., $\left.1 \mathrm{H}, \mathrm{OH}\right), 1.43-1.33$ (m, $2 \mathrm{H}, \mathrm{CH}_{2}$ ), $1.28-1.15\left(\mathrm{~m}, 10 \mathrm{H}, 5 \mathrm{CH}_{2}\right), 0.81\left(\mathrm{t}, J=7 \mathrm{~Hz}, 3 \mathrm{H}, \mathrm{CH}_{3}\right)$; $\delta_{\mathrm{C}}\left(\mathrm{CDCl}_{3}\right) 135.3\left(\mathrm{~d}, \mathrm{CH}=\mathrm{CH}_{2}\right), 118.5\left(\mathrm{t}, \mathrm{CH}=\mathrm{CH}_{2}\right), 71.1(\mathrm{~d}$, $\mathrm{CHOH}), 41.3\left(\mathrm{t}, \mathrm{CH}_{2}\right), 37.2\left(\mathrm{t}, \mathrm{CH}_{2}\right), 33.2\left(\mathrm{t}, \mathrm{CH}_{2}\right), 30.0(\mathrm{t}$ $\left.\mathrm{CH}_{2}\right), 29.7\left(\mathrm{t}, \mathrm{CH}_{2}\right), 26.1\left(\mathrm{t}, \mathrm{CH}_{2}\right), 23.1\left(\mathrm{t}, \mathrm{CH}_{2}\right), 14.5\left(\mathrm{q}, \mathrm{CH}_{3}\right)$.

\section{1-Cyclohexylbut-3-en-1-ol (3I) ${ }^{28}$}

Oil, $\delta_{\mathrm{H}}\left(\mathrm{CDCl}_{3}\right)$ 5.82-5.71 (m, 1 H, $\left.\mathrm{CH}=\mathrm{CH}_{2}\right), 5.11-5.04(\mathrm{~m}$, $\left.2 \mathrm{H}, \mathrm{CH}=\mathrm{CH}_{2}\right), 3.35-3.29(\mathrm{~m}, 1 \mathrm{H}, \mathrm{CHOH}), 2.30-2.01(\mathrm{~m}, 2 \mathrm{H}$ $\mathrm{CH}_{2}$ ), 1.78 (br s, exch., $\left.1 \mathrm{H}, \mathrm{OH}\right), 1.70-1.55$ (m, $5 \mathrm{H}$, cyclohexyl), 1.34-0.90 (m, 6 H, cyclohexyl); $\delta_{\mathrm{C}}\left(\mathrm{CDCl}_{3}\right) 135.9(\mathrm{~d}$, $\left.\mathrm{CH}=\mathrm{CH}_{2}\right), 118.4\left(\mathrm{t}, \mathrm{CH}=\mathrm{CH}_{2}\right), 75.1(\mathrm{~d}, \mathrm{CHOH}), 43.5\left(\mathrm{t}, \mathrm{CH}_{2}\right)$, $39.2(\mathrm{~d}, \mathrm{CH}), 29.5\left(\mathrm{t}, \mathrm{CH}_{2}\right), 28.5\left(\mathrm{t}, \mathrm{CH}_{2}\right), 26.9\left(\mathrm{t}, \mathrm{CH}_{2}\right), 26.7(\mathrm{t}$ $\left.\mathrm{CH}_{2}\right), 26.5\left(\mathrm{t}, \mathrm{CH}_{2}\right)$.

\section{Acknowledgements}

We thank the EPSRC and the University of Wales Swansea for financial support. We also thank the EPSRC, the Higher Education Funding Council for Wales (ELWa-HEFCW) and the University of Wales Swansea for grants that enabled the purchase and upgrading of NMR equipment used in the course of this work. G. A. El-Hiti thanks the Royal Society of Chemistry for an international author grant.

\section{References}

1 C. Blomberg and F. A. Hartog, Synthesis, 1977, 18.

2 C.-J. Li, Tetrahedron, 1996, 52, 5643.

3 P. T. Anastas and R. Lankey, Chem. Br, 2001, 37, 26

4 A. Lubineau, J. Augé and Y. Queneau, Synthesis, 1994, 741.

5 P. C. Andrews and C. L. Raston, J. Organomet. Chem., 2000, 600, 174.

6 M. Wada, H. Ohki and K. Akiba, Bull. Chem. Soc. Jpn., 1990, 63, 1738.

7 H. Suzuki, T. Ikegami and Y. Matano, Synthesis, 1997, 249.

8 P. J. Sadler, H. Li and H. Sun, Coord. Chem. Rev., 1999, 185, 689.

9 T.-H. Chan and C.-J. Li, Organometallics, 1990, 9, 2649.
10 C.-J. Li and T.-H. Chan, Organometallics, 1991, 10, 2548

11 T.-H. Chan and C.-J. Li, Can. J. Chem., 1992, 70, 2726.

12 M. Lombardo, R. Girotti, S. Morganti and C. Trombini, Chem. Commun., 2001, 2310.

13 C. Petrier and J.-L. Luche, J. Org. Chem., 1985, 50, 910.

14 C. Petrier, J. Einhorn and J.-L. Luche, Tetrahedron Lett., 1985, 26, 1445.

15 C. Einhorn and J.-L. Luche, J. Organomet. Chem., 1987, 322, 177.

16 S. R. Wilson and M. E. Guazzaroni, J. Org. Chem., 1989, 54, 3087.

17 R. Sjoholm, R. Rairama and M. Ahonen, J. Chem. Soc.,Chem. Commun., 1994, 1217.

18 M. Wada and K. Akiba, Tetrahedron Lett., 1985, 26, 4211

19 N. Miyoshi, M. Nishio, S. Murakami, T. Fukuma and M. Wada, Bull. Chem. Soc. Jpn., 2000, 73, 689.

20 P. C. Andrews, A. C. Peatt and C. L. Raston, Green Chem., 2001, 3, 313.

21 S. Wada, N. Hayashi and H. Suzuki, Org. Biomol. Chem., 2003, 1, 2160 .

22 See for example; K. Smith, G. A. El-Hiti, M. A. Abdo and M. F. Abdel-Megeed, J. Chem. Soc., Perkin Trans. 1, 1995, 1029; K. Smith, G. A. El-Hiti, M. F. Abdel-Megeed and M. A. Abdo, J. Org. Chem., 1996, 61, 647-656; K. Smith, G. A. El-Hiti and A. Hamilton, J. Chem. Soc., Perkin Trans. 1, 1998, 4041; K. Smith, G. A. El-Hiti, G. J. Pritchard and A. Hamilton, J. Chem. Soc., Perkin Trans. 1, 1999, 2299; K. Smith, G. A. El-Hiti and A. P. Shukla, J. Chem. Soc., Perkin Trans. 1, 1999, 2305; K. Smith, G. A. El-Hiti and M. F. Abdel-Megeed, Russ. J. Org. Chem., 2003, 39, 430; K. Smith, G. A. El-Hiti and A. C. Hawes, Synthesis, 2003, 2047; K. Smith, G. A. El-Hiti and S. A. Mahgoub, Synthesis, 2003, 2345.

23 See for example; K. Smith, A. Musson and G. A. DeBoos, J. Org. Chem., 1998, 63, 8448; K. Smith, Z. Zhenhua and P. K. G. Hodgson, J. Mol. Catal. A, 1998, 134, 121; K. Smith, P. He and A. Taylor, Green Chem., 1999, 1, 35; K. Smith, M. Butters, W. E. Paget, D. Goubet, E. Fromentin and B. Nay, Green Chem., 1999, 1, 83; K. Smith, T. Gibbins, R. W. Millar and R. P. Claridge, J. Chem. Soc., Perkin Trans. 1, 2000, 2753; K. Smith, G. A. El-Hiti, M. E. W. Hammond, D. Bahzad, Z. Li and C. Siquet, J. Chem. Soc., Perkin Trans. 1, 2000, 2745; K. Smith, S. Almeer, S. J. Black and C. Peters, J. Mater. Chem., 2002, 12, 3285; K. Smith, S. D. Roberts and G. A. El-Hiti, Org. Biomol. Chem., 2003, 1, 1552; K. Smith, G. A. El-Hiti, A. Jayne and M. Butters, Org. Biomol. Chem., 2003, 1, 1560-2321.

24 L.-H. Li and T. H. Chan, Tetrahedron Lett., 2000, 41, 5009

25 L.-H. Li and T. H. Chan, Org. Lett., 2000, 2, 1129.

26 Y.-Z. Huang and Y. Liao, J. Org. Chem., 1991, 56, 1381.

27 A. Hosomi, S. Kohra, K. Ogata, T. Yanagi and Y. Tominaga, J. Org. Chem. 1990, 55, 2415.

28 A. P. Davis and M. Jaspars, J. Chem. Soc., Perkin Trans. 1, 1992, 2111.

29 R. A. Batey, A. N. Thadani, D. V. Smil and A. J. Lough, Synthesis, $2000,990$.

30 Q. Zhang, Z. Luo and D. P. Curran, J. Org. Chem., 2000, 65, 8866.

31 I. Shibata, N. Yoshimura, M. Yabu and A. Baba, Eur. J. Org. Chem., 2001, 3207

32 V. K. Aggarwal and G. P. Vennall, Synthesis, 1998, 1822. 